TRANSACTIONS OF THE

AMERICAN MATHEMATICAL SOCIETY

Volume 357, Number 2, Pages 837-850

S 0002-9947(04)03549-4

Article electronically published on July 22, 2004

\title{
HARNACK INEQUALITIES FOR NON-LOCAL OPERATORS OF VARIABLE ORDER
}

\author{
RICHARD F. BASS AND MORITZ KASSMANN
}

AbSTRACT. We consider harmonic functions with respect to the operator

$$
\mathcal{L} u(x)=\int\left[u(x+h)-u(x)-1_{(|h| \leq 1)} h \cdot \nabla u(x)\right] n(x, h) d h .
$$

Under suitable conditions on $n(x, h)$ we establish a Harnack inequality for functions that are nonnegative and harmonic in a domain. The operator $\mathcal{L}$ is allowed to be anisotropic and of variable order.

\section{INTRODUCTION}

There is a huge literature concerned with Harnack inequalities for functions that are harmonic with respect to second order elliptic operators. Seminal contributions in this field have been made among others by Moser Mos61, Krylov-Safonov KS80, and Fabes-Stroock [FS86. The first and third of these papers deal with differential operators in divergence form, while the second deals with differential operators in non-divergence form. These papers, as well as alternate proofs of their results, all rely heavily on the fact that the operators are local operators, that is, differential operators.

At the same time, in the last few years there has been intense interest in using integral operators (or equivalently, processes with jumps) to model problems in mathematical physics, in finance, and in probability theory. These operators are non-local, in the sense that the behavior of a harmonic function at a point depends on values of the harmonic function at points some distance away rather than just at nearby points.

The purpose of this paper is to consider functions that are harmonic with respect to the integral operator $\mathcal{L}$, where

$$
\mathcal{L} u(x)=\int_{\mathbb{R}^{d} \backslash\{0\}}\left[u(x+h)-u(x)-1_{(|h| \leq 1)} h \cdot \nabla u(x)\right] n(x, h) d h
$$

operates on $C^{2}$ functions defined on $\mathbb{R}^{d}$. This is a reasonably general integrodifferential operator, and includes, for example, many of the operators considered by probabilists. In probabilistic terms, $n(x, h)$ represents the relative intensity of the number of jumps of the associated Markov process from a point $x$ to the point $x+h$. We examine what conditions are needed on $n(x, h)$ to guarantee that

Received by the editors May 27, 2003 and, in revised form, October 27, 2003.

2000 Mathematics Subject Classification. Primary 45K05; Secondary 60H10.

Key words and phrases. Harnack inequality, non-local operator, stable processes, Lévy processes, jump processes, integral operators.

The first author's research was partially supported by NSF grant DMS-9988496. 
a Harnack inequality holds. We start with the assumption that for two positive constants $\kappa_{1}$ and $\kappa_{2}$

$$
\frac{\kappa_{1}}{|h|^{d+\alpha}} \leq n(x, h) \leq \frac{\kappa_{2}}{|h|^{d+\beta}}, \quad x \in \mathbb{R}^{d}, \quad|h| \leq 2,
$$

where $0<\alpha<\beta<2$. This is the analogue of the coercivity and boundedness conditions from the theory of elliptic PDE. Note that the order of the singularity of the kernel with respect to $h$ might depend on $x$. Moreover, the kernel might exhibit different singularities in different directions. Hence, the corresponding integrodifferential operator $\mathcal{L}$ is anisotropic and of variable order. For now let us say that a function $u$ is harmonic with respect to $\mathcal{L}$ in a domain $D$ if $\mathcal{L} u=0$ in $D$; a more precise definition is given in Section 2 in terms of martingales.

Our main result is that if $\beta-\alpha<1$, then a Harnack inequality holds for nonnegative functions that are harmonic in a domain; see Theorem 4.1 for a precise statement. We do not know if our condition $\beta-\alpha<1$ is sharp. The conclusion of Theorem 4.1 says that $u(x) \leq \bar{\kappa}(R) u(y)$ for $x, y$ in a ball of radius $R / 2$ when $u$ is harmonic in the concentric ball of radius $R$. In Proposition 5.1] we give an example to show that the dependence of $\bar{\kappa}$ on $R$ cannot be dispensed with.

At the time of the writing of this paper, there are only a few papers that we know of that consider Harnack inequalities for non-local operators. In [BBG00] a very specific operator was considered; there the interest was not in the Harnack inequality but in a Liouville property for a certain degenerate PDE. In [BL02a] the operator $\mathcal{L}$ given in (1.1) was considered, but in the special case where $\alpha=\beta$, which is sometimes known as the stable-like case. The results of [BL02a were extended to certain other Markov jump processes in [SV04]. A parabolic Harnack inequality for symmetric jump processes, again with $\alpha=\beta$, together with heat kernel estimates, was proved in BL02b. This was extended to more general state spaces in CK03. See [BSS02a and [BSS02b] for related results. A weak Harnack inequality has been obtained in Kas03 for non-local operators corresponding to jump-diffusions.

The current paper is a major generalization of the results obtained in BL02a] and SV04] in that we remove the requirement $\alpha=\beta$. We are able to allow the integro-differential operators to be anisotropic and of variable order.

The method starts with the ideas of [BL02a], but due to the fact that $\alpha \neq \beta$, the techniques are considerably more delicate. Both [BL02a] and the current paper use techniques substantially different from those used in the case of elliptic operators, although the roots of our method come from those of [KS80]. It is interesting that while in [KS80] the hardest part of the proof is obtaining what is essentially an estimate on the probability of hitting sets; here, by contrast, the corresponding estimate is fairly easy. The principal difficulty in this paper is using that estimate to obtain the Harnack inequality.

After a short section on preliminaries, in Section 3 we present some estimates for the Markov process associated with $\mathcal{L}$. These are used in Section 4 to prove the Harnack inequality. Section 5 contains some examples.

\section{Preliminaries}

We use $B(x, r)$ for the open ball of radius $r$ with center $x$. The letter $c$ with subscripts will denote positive finite constants whose exact value is unimportant. The Lebesgue measure of a Borel set $A$ will be denoted by $|A|$. 
We consider the operator

$$
\mathcal{L} u(x)=\int_{h \neq 0}\left[u(x+h)-u(x)-1_{(|h| \leq 1)} h \cdot \nabla u(x)\right] n(x, h) d h .
$$

Suppose $0<\alpha<\beta<2$. We make the following assumptions on $n(x, h)$.

Assumption 2.1. There exist positive finite constants $\kappa_{1}, \kappa_{2}, \kappa_{3}, \kappa_{4}$ such that:

- (a) For all $|h| \leq 2$ and all $x$

$$
n(x, h) \geq \frac{\kappa_{1}}{|h|^{d+\alpha}} .
$$

- (b) For all $|h| \leq 2$ and all $x$

$$
n(x, h) \leq \frac{\kappa_{2}}{|h|^{d+\beta}} .
$$

- (c) For all $x$

$$
\int_{|h|>1} n(x, h) d h \leq \kappa_{3} .
$$

- (d) For all $x, y$, and $z$

$$
n(x, z-x) \leq \kappa_{4} n(y, z-y), \quad|z-x| \geq 1,|z-y| \geq 1,|x-y| \leq 1 .
$$

Assumptions 2.1 (a)-(c) say that the Lévy kernel $n(x, h) d h$ is bounded between that of a symmetric stable process of index $\alpha$ and that of one of index $\beta$ for the jumps of size less than 2. Moreover, we have a uniform bound on the number of jumps of size bigger than 1. $n(x, h)$ can be thought of as the intensity of the number of jumps from $x$ to $x+h$; thus $n(x, z-x)$ represents the intensity of the number of jumps from $x$ to $z$. Assumption 2.1 (d) says that the probability of jumping to a point $z$ is comparable if $x, y$ are more than distance one away from $z$ and within distance one of each other. Note that the constant "one" could be replaced by another appropriate positive constant. In Proposition 5.2 we show that an assumption of this type cannot be avoided.

Our method is probabilistic, and we need to work with the Markov process associated with $\mathcal{L}$. We say a strong Markov process $\left(\mathbb{P}^{x}, X_{t}\right)$ is associated with $\mathcal{L}$ if for each $x$ we have $\mathbb{P}^{x}\left(X_{0}=x\right)=1$ and for each $x$ and for each $u \in C^{2}$ that is bounded with bounded first and second partial derivatives, $u\left(X_{t}\right)-u\left(X_{0}\right)-$ $\int_{0}^{t} \mathcal{L} u\left(X_{s}\right) d s$ is a martingale under $\mathbb{P}^{x}$. This is commonly expressed as saying that $\mathbb{P}^{x}$ solves the martingale problem for $\mathcal{L}$ started at $x$.

Without some regularity on $n(x, h)$ we do not know that there is a strong Markov process associated with $\mathcal{L}$ or that if there is one, it is unique. One of the major open problems in the area of uniqueness is to formulate simple but not too restrictive sufficient conditions. If $n(x, h)$ depends on $x$ in a Lipschitz fashion, it is known that uniqueness holds, see [Sko65]. Let us assume that $n(x, h)$ satisfies some conditions (see Kom84, Bas88, Hoh95, Sko65]) which insure that there is one and only one solution to the martingale problem for $\mathcal{L}$ started at $x$. However, we underline that none of the constants in any of our results depend on the smoothness or regularity of $n(x, h)$.

An equivalent formulation of the connection between the Markov process and the operator $\mathcal{L}$ can be made in terms of a stochastic differential equation driven by a random measure, but this is less direct. 
For any Borel set $A$, let

$$
T_{A}=\inf \left\{t: X_{t} \in A\right\}, \quad \tau_{A}=\inf \left\{t: X_{t} \notin A\right\},
$$

the first hitting time and first exit time, respectively, of $A$. We say that a function $u$ is harmonic in a domain $D$ if $u\left(X_{t \wedge \tau_{D}}\right)$ is a $\mathbb{P}^{x}$-martingale for each $x \in D$. It is easy to check that if $u$ satisfies some smoothness conditions (e.g., $u$ and its first and second partials are bounded and continuous in $D$ ) and $\mathcal{L} u=0$ in $D$, then $u$ is harmonic in $D$.

Similarly to the diffusion case explained in SV79], Corollary 6.3.3, uniqueness of solutions to the martingale problem imply that the corresponding process is a Feller process. Therefore our Markov process is a Hunt process (see BG68, Section 1.9) and in particular left hand limits exist. We write $X_{t-}=\lim _{s \uparrow t} X_{s}$ and $\Delta X_{t}=X_{t}-X_{t-}$. Any harmonic function $u$ is excessive with respect to the semigroup of $X_{t}$ and therefore $u\left(X_{t \wedge \tau_{D}}\right)$ is right-continuous, with the exceptional set having $\mathbb{P}^{x}$-measure zero for all $x$; see BG68, Theorem II.2.12.

\section{Some estimates}

Throughout this section we assume that Assumption 2.1 holds. Set

$$
\bar{\beta}=\max (\beta, 1) \text {. }
$$

Proposition 3.1. There exist constants $c_{1}$ and $c_{2}$ not depending on $x_{0}$ such that if $r<1, \beta \neq 1$ and $t>0$, then

$$
\mathbb{P}^{x_{0}}\left(\tau_{B\left(x_{0}, r\right)} \leq c_{1} t\right) \leq t r^{-\bar{\beta}},
$$

and in particular

$$
\mathbb{P}^{x_{0}}\left(\tau_{B\left(x_{0}, r\right)} \leq c_{2} r^{\bar{\beta}}\right) \leq \frac{1}{2} .
$$

Proof. Let $u$ be a nonnegative $C^{2}$ function that is equal to $\left|x-x_{0}\right|^{2}$ for $\left|x-x_{0}\right| \leq r / 2$, which equals $r^{2}$ for $\left|x-x_{0}\right| \geq r$, and such that $u$ is bounded by $c_{3} r^{2}$, its first partial derivatives are bounded by $c_{3} r$, and its second partial derivatives are bounded by $c_{3}$. Then, since $\mathbb{P}^{x_{0}}$ solves the martingale problem,

$$
\mathbb{E}^{x_{0}} u\left(X_{\left.t \wedge \tau_{B\left(x_{0}, r\right)}\right)}\right)-u\left(x_{0}\right)=\mathbb{E}^{x_{0}} \int_{0}^{t \wedge \tau_{B\left(x_{0}, r\right)}} \mathcal{L} u\left(X_{s}\right) d s .
$$

We examine $\mathcal{L} u(x)$ for $x \in B\left(x_{0}, r\right)$. We break the integral in (2.1) into two parts, where $|h| \leq r$ and where $|h|>r$. For the first part, we have

$$
\int_{|h| \leq r}[u(x+h)-u(x)-h \cdot \nabla u(x)] n(x, h) d h \leq c_{4} \int_{|h| \leq r} h^{2} n(x, h) d h,
$$

since the expression inside the brackets is bounded by a constant times $h^{2}\left\|D^{2} u\right\|_{\infty}$. Since for $|h| \leq r$ we have $n(x, h) \leq c_{5} h^{-d-\beta}$, we bound the above by $c_{6} r^{2-\beta}$. For the second part we obtain, using Assumptions 2.1 (b) and (c),

$$
\int_{|h|>r}[u(x+h)-u(x)] n(x, h) d h \leq\|u\|_{\infty} \int_{|h|>r} n(x, h) d h \leq c_{7} r^{2-\beta},
$$

and, using $\|\nabla u\|_{\infty} \leq c_{2} r$,

$$
\left|\int_{1>|h| \geq r} h \cdot \nabla u(x) n(x, h) d h\right| \leq c_{8} r^{2-\bar{\beta}} .
$$


Substituting in (3.2), we obtain

$$
\mathbb{E}^{x_{0}} u\left(X_{t \wedge \tau_{B\left(x_{0}, r\right)}}\right) \leq c_{9} t r^{2-\bar{\beta}} .
$$

Note that the left hand side is greater than $r^{2} \mathbb{P}^{x_{0}}\left(\tau_{B\left(x_{0}, r\right)} \leq t\right)$, which yields the first part of the proposition. If we now take $t=c_{10} r^{\bar{\beta}}$, we obtain the second part of the proposition.

Proposition 3.2. If $A$ and $B$ are disjoint Borel sets, then for each $x$

$$
\sum_{s \leq t} 1_{\left(X_{s-} \in A, X_{s} \in B\right)}-\int_{0}^{t} \int_{B} 1_{A}\left(X_{s}\right) n\left(X_{s}, u-X_{s}\right) d u d s
$$

is a $\mathbb{P}^{x}$-martingale.

The proof is identical to that of Proposition 2.3 and Remark 2.4 of [BL02a].

The next proposition estimates the hitting probability for certain sets. It is notable that, unavoidably, the conclusion is considerably weaker than that of Theorem 1 in [KS79]; namely, the hitting probability is not bounded away from zero. Despite this difference from the non-degenerate diffusion case, we are able to prove a Harnack inequality.

Proposition 3.3. Suppose $r<1$ and $\beta \neq 1$.

- (a) There exists $c_{1}$ such that if $A \subset B\left(x_{0}, r / 2\right)$ and also $y \in B\left(x_{0}, r / 2\right)$, then

$$
\mathbb{P}^{y}\left(T_{A}<\tau_{B\left(x_{0}, r\right)}\right) \geq c_{1} r^{\bar{\beta}-\alpha}|A| /\left|B\left(x_{0}, r\right)\right| .
$$

- (b) There exists $c_{1}$ such that if $A \subset B\left(x_{0}, r / 2\right)$ and also $y \in B\left(x_{0}, r\right)$, then

$$
\mathbb{P}^{y}\left(T_{A}<\tau_{B\left(x_{0}, r\right)}\right) \geq c_{1}\left[\operatorname{dist}\left(y, \partial B\left(x_{0}, r\right)\right)\right]^{\bar{\beta}} r^{-\alpha}|A| /\left|B\left(x_{0}, r\right)\right| .
$$

Proof. (a) is an immediate consequence of (b), so we prove (b). Fix $y$ and write $\tau$ for $\tau_{B\left(x_{0}, r\right)}$. Let $p=\operatorname{dist}\left(y, \partial B\left(x_{0}, r\right)\right)$. If $\mathbb{P}^{y}\left(T_{A}<\tau\right) \geq \frac{1}{4}$, we are done, so we assume not. By Proposition 3.1 we can find a constant $c_{2}$ such that if $t_{0}=c_{2} p^{\bar{\beta}}$, then $\mathbb{P}^{y}\left(\tau \leq t_{0}\right) \leq \frac{1}{2}$. If $x \in B\left(x_{0}, r\right)$ and $z \in A$, then $|z-x| \leq 2 r$ and

$$
n(x, z-x) \geq c_{3}|z-x|^{-d-\alpha} \geq c_{4} r^{-d-\alpha} .
$$

Then by Proposition 3.2 and optional stopping,

$$
\begin{aligned}
\mathbb{P}^{y}\left(T_{A}<\tau\right) & \geq \mathbb{E}^{y} \sum_{s \leq T_{A} \wedge \tau \wedge t_{0}} 1_{\left(X_{s-} \neq X_{s}, X_{s} \in A\right)} \\
& =\mathbb{E}^{y} \int_{0}^{T_{A} \wedge \tau \wedge t_{0}} \int_{A} n\left(X_{s}, z-X_{s}\right) d z d s \\
& \geq c_{4}|A| r^{-d-\alpha} \mathbb{E}^{y}\left(T_{A} \wedge \tau \wedge t_{0}\right) .
\end{aligned}
$$

We also have

$$
\begin{aligned}
\mathbb{E}^{y}\left(T_{A} \wedge \tau \wedge t_{0}\right) & \geq \mathbb{E}^{y}\left(t_{0} ; T_{A} \geq \tau \geq t_{0}\right)=t_{0} \mathbb{P}^{y}\left(T_{A} \geq \tau \geq t_{0}\right) \\
& \geq t_{0}\left[1-\mathbb{P}^{y}\left(T_{A}<\tau\right)-\mathbb{P}^{y}\left(\tau<t_{0}\right)\right] \geq t_{0} / 4
\end{aligned}
$$

Therefore

$$
\mathbb{P}^{y}\left(T_{A}<\tau\right) \geq \frac{c_{4}}{4}|A| r^{-d-\alpha} t_{0}=c_{5} p^{\bar{\beta}} r^{-\alpha}|A| /\left|B\left(x_{0}, r\right)\right| .
$$


Lemma 3.4. There exist $c_{1}$ and $c_{2}$ such that if $r<1 / 2$ and $\beta \neq 1$, then

$$
\mathbb{E}^{x} \tau_{B(x, r)} \geq c_{1} r^{\bar{\beta}}, \quad \mathbb{E}^{x} \tau_{B(x, r)} \leq c_{2} r^{\alpha} .
$$

Proof. By Proposition 3.1 there exists $c_{3}$ such that $\mathbb{P}^{x}\left(\tau_{B(x, r)} \leq c_{3} r^{\bar{\beta}}\right) \leq \frac{1}{2}$. So $\tau_{B(x, r)}$ is greater than $c_{3} r^{\bar{\beta}}$ with probability at least $\frac{1}{2}$, and the first inequality follows easily from this.

To prove the second inequality, let $S$ be the time of the first jump larger than $2 r$. Suppose $\mathbb{P}^{z}\left(S \leq r^{\alpha}\right) \leq \frac{1}{2}$. Then by Proposition 3.2 and optional stopping,

$$
\begin{aligned}
\mathbb{P}^{z}\left(S \leq r^{\alpha}\right) & =\mathbb{E}^{z} \sum_{s \leq S \wedge r^{\alpha}} 1_{\left(\left|X_{s}-X_{s-}\right|>2 r\right)}=\mathbb{E}^{z} \int_{0}^{S \wedge r^{\alpha}} \int_{|h|>2 r} n\left(X_{s}, h\right) d h d s \\
& \geq \mathbb{E}^{z} \int_{0}^{S \wedge r^{\alpha}} \int_{2 \geq|h|>2 r} n\left(X_{s}, h\right) d h d s \\
& \geq c_{4} r^{-\alpha} \mathbb{E}^{z}\left(S \wedge r^{\alpha}\right) \geq c_{4} r^{-\alpha} \mathbb{E}^{z}\left(r^{\alpha} ; S>r^{\alpha}\right) \\
& \geq c_{4} \mathbb{P}^{z}\left(S>r^{\alpha}\right) \geq c_{4} / 2 .
\end{aligned}
$$

The other alternative is that $\mathbb{P}^{z}\left(S \leq r^{\alpha}\right)>\frac{1}{2}$. In either case there exists $c_{5}$ such that $\mathbb{P}^{z}\left(S \leq r^{\alpha}\right) \geq c_{5}>0$.

If $\theta_{t}$ is the shift operator from Markov process theory, then by the Markov property

$$
\begin{aligned}
\mathbb{P}^{z}\left(S>(m+1) r^{\alpha}\right) & \leq \mathbb{P}^{z}\left(S>m r^{\alpha}, S \circ \theta_{m r^{\alpha}}>r^{\alpha}\right) \\
& =\mathbb{E}^{z}\left[\mathbb{P}^{X_{m r^{\alpha}}}\left(S>r^{\alpha}\right) ; S>m r^{\alpha}\right] \\
& \leq\left(1-c_{5}\right) \mathbb{P}^{z}\left(S>m r^{\alpha}\right) .
\end{aligned}
$$

By induction $\mathbb{P}^{z}\left(S>m r^{\alpha}\right) \leq\left(1-c_{5}\right)^{m}$, which proves $\mathbb{E}^{x} S \leq c_{6} r^{\alpha}$. Our second inequality follows because $\tau_{B(x, r)} \leq S$ when we start the process at $x$.

Proposition 3.5. There exists $c_{1}$ such that if $r<1 / 2, \beta \neq 1, z \in B(x, r / 4)$, and $H$ is a bounded nonnegative function supported in $B(x, r)^{c}$, then

$$
\mathbb{E}^{x} H\left(X_{\tau_{B(x, r / 2)}}\right) \leq c_{1} r^{2(\alpha-\bar{\beta})} \mathbb{E}^{z} H\left(X_{\tau_{B(x, r / 2)}}\right) .
$$

Proof. By linearity and a limit argument, it suffices to consider $H=1_{C}$ for a set $C$ contained in $B(x, r)^{c}$. Note that Assumptions 2.1 (a), (b) and (d) imply that if $v \notin B(x, r)$, then

$$
\sup _{y \in B(x, r / 2)} n(y, v-y) \leq c_{2} r^{\alpha-\bar{\beta}} \inf _{y \in B(x, r / 2)} n(y, v-y) .
$$

Write $\tau$ for $\tau_{B(x, r / 2)}$. For $X_{\tau}$ to be in $C$, it must get there by a jump of size at least $r / 2$. By Proposition 3.2 and optional stopping,

$$
\begin{aligned}
\mathbb{E}^{z} 1_{\left(X_{t \wedge \tau} \in C\right)} & =\mathbb{E}^{z} \sum_{s \leq t \wedge \tau} 1_{\left(\left|X_{s-}-X_{s}\right|>r / 2, X_{s} \in C\right)} \\
& =\mathbb{E}^{z} \int_{0}^{t \wedge \tau} \int_{C} n\left(X_{s}, v-X_{s}\right) d v d s \\
& \geq\left(\mathbb{E}^{z}(t \wedge \tau)\right)\left(\int_{C} \inf _{y \in B(x, r / 2)} n(y, v-y) d v\right) .
\end{aligned}
$$


Letting $t \rightarrow \infty$ and using dominated convergence on the left and monotone convergence on the right, we get

$$
\mathbb{P}^{z}\left(X_{\tau} \in C\right) \geq \mathbb{E}^{z} \tau \int_{C} \inf _{y \in B(x, r / 2)} n(y, v-y) d v .
$$

Since $\mathbb{E}^{z} \tau \geq \mathbb{E}^{z} \tau_{B(z, r / 4)}$, Lemma 3.4 tells us that

$$
\mathbb{P}^{z}\left(X_{\tau} \in C\right) \geq c_{3} r^{\bar{\beta}} \int_{C} \inf _{y \in B(x, r / 2)} n(y, v-y) d v .
$$

Similarly,

$$
\mathbb{P}^{x}\left(X_{\tau} \in C\right) \leq \mathbb{E}^{x} \tau \int_{C} \sup _{y \in B(x, r / 2)} n(y, v-y) d v .
$$

Lemma 3.4 (3.3), (3.4), and (3.5) then imply our result.

\section{HARNACK INEQUALITY}

Theorem 4.1. Suppose Assumption 2.1 holds. Suppose $\beta-\alpha<1$. Let $z_{0} \in \mathbb{R}^{d}$ and $R>0$. Suppose $u$ is nonnegative and bounded on $\mathbb{R}^{d}$ and harmonic on $B\left(z_{0}, R\right)$. Then there exists a constant $\bar{\kappa}$ depending on $R, \kappa_{1}, \kappa_{2}, \kappa_{3}, \kappa_{4}$ but not $z_{0}, u$, or $\|u\|_{\infty}$ such that

$$
u(x) \leq \bar{\kappa} u(y), \quad x, y \in B\left(z_{0}, R / 2\right) .
$$

Proof. Since $\beta-\alpha<1$ and $\alpha>0$, we can take $\beta$ bigger if necessary so that $\beta>1$ and $\beta-\alpha<1$. So without loss of generality we may assume $\beta>1$, and hence $\bar{\beta}=\beta$.

Let us first suppose $R \leq 1$. By looking at $u+\varepsilon$ and letting $\varepsilon \downarrow 0$, we may suppose $u$ is bounded below by a positive constant. By looking at $a u$ for a suitable constant $a$, we may suppose $\inf _{B\left(z_{0}, R / 2\right)} u \in\left[\frac{1}{2}, 1\right]$. (We do not know that $u$ is continuous, so the infimum might not be attained.) We want to bound $u$ above in $B\left(z_{0}, R / 2\right)$ by a constant depending only on $R$ and $\kappa_{1}, \kappa_{2}, \kappa_{3}, \kappa_{4}$. Choose $z_{1} \in B\left(z_{0}, R / 2\right)$ such that $u\left(z_{1}\right) \leq 1$. Choose $\rho$ such that $1<\rho<1 /(\beta-\alpha)$.

Let

$$
r_{i}=c_{2} R / i^{\rho},
$$

where $c_{2}$ is a constant that will be chosen later. We require first of all that $c_{2}$ be small enough so that

$$
\sum_{i=1}^{\infty} r_{i} \leq R / 8
$$

which, in particular, implies $r_{i} \leq 1 / 8$.

Recall that by Proposition 3.3(b) there exists $c_{3}$ such that for any $\bar{z}, \bar{r}, A \subset$ $B(\bar{z}, \bar{r} / 2)$ and $\bar{x} \in B(\bar{z}, \bar{r} / 2)$ we have

$$
\mathbb{P}^{\bar{x}}\left(T_{A}<\tau_{B(\bar{z}, \bar{r})}\right) \geq c_{3} \bar{r}^{\beta-\alpha}|A| /|B(\bar{z}, \bar{r} / 2)| .
$$

Let $c_{4}$ be another constant to be chosen later. Once $c_{2}$ and $c_{4}$ have been chosen, choose $K_{1}$ sufficiently large so that

$$
\frac{1}{4} c_{3} K_{1} \exp \left(R c_{2} c_{4} i^{1-\rho(\beta-\alpha)}\right) c_{2}^{5(\beta-\alpha)+d} R^{5(\beta-\alpha)} \geq 2 i^{\rho(5(\beta-\alpha)+d)}
$$

for $i=1,2, \ldots$. Such a choice is possible because $1-\rho(\beta-\alpha)>0 . K_{1}$ will depend on $d, R, \rho, \alpha$, and $\beta$ as well as $c_{2}, c_{3}$ and $c_{4}$. 
Suppose now that there exists $x_{1} \in B\left(z_{0}, R / 2\right)$ with $u\left(x_{1}\right) \geq K_{1}$. We will show that in this case there exists a sequence $\left\{\left(x_{j}, K_{j}\right)\right\}$ with $x_{j+1} \in B\left(x_{j}, 2 r_{j}\right) \subset$ $B\left(z_{0}, 3 R / 4\right), K_{j}=u\left(x_{j}\right)$, and

$$
K_{j} \geq K_{1} \exp \left(R c_{2} c_{4} j^{1-\rho(\beta-\alpha)}\right) .
$$

Since $1-\rho(\beta-\alpha)>0$, then $K_{j} \rightarrow \infty$, a contradiction to $u$ being bounded. We can then conclude that $u$ must be bounded by $K_{1}$, and hence $u(x) / u(y) \leq 2 K_{1}$ if $x, y \in B\left(z_{0}, R / 2\right)$.

Suppose $x_{1}, x_{2}, \ldots, x_{i}$ have been selected and that (4.5) holds for $j=1, \ldots, i$. We will show there exists $x_{i+1} \in B\left(x_{i}, 2 r_{i}\right)$ such that if $K_{i+1}=u\left(x_{i+1}\right)$, then (4.5) holds for $j=i+1$; we then use induction to conclude that (4.5) holds for all $j$.

Let

$$
A_{i}=\left\{y \in B\left(x_{i}, r_{i} / 4\right): u(y) \geq K_{i} r_{i}^{4(\beta-\alpha)}\right\} .
$$

First, we prove that

$$
\left|A_{i}\right| /\left|B\left(x_{i}, r_{i} / 4\right)\right| \leq \frac{1}{4}
$$

To prove this claim, we suppose to the contrary that $\left|A_{i}\right| /\left|B\left(x_{i}, r_{i} / 4\right)\right|>\frac{1}{4}$. Let $D$ be a compact subset of $A_{i}$ with $|D| /\left|B\left(x_{i}, r_{i} / 4\right)\right|>\frac{1}{4}$. Recall that $R \geq 8 r_{i} \geq r_{i}$. By Doob's optional stopping theorem, the facts that $u$ is nonnegative and $u\left(X_{t \wedge \tau_{D}}\right)$ is right-continuous, (4.3), (4.4), and (4.5), we can estimate

$$
\begin{aligned}
1 & \geq u\left(z_{1}\right) \geq \mathbb{E}^{z_{1}}\left[u\left(X_{\left.T_{D} \wedge \tau_{B\left(z_{0}, R\right)}\right)}\right) T_{D}<\tau_{B\left(z_{0}, R\right)}\right] \\
& \geq K_{i} r_{i}^{4(\beta-\alpha)} \mathbb{P}^{z_{1}}\left(T_{D}<\tau_{B\left(z_{0}, R\right)}\right) \\
& \geq c_{3} K_{i} r_{i}^{4(\beta-\alpha)} R^{\beta-\alpha}|D| /\left|B\left(z_{0}, R\right)\right| \\
& \geq \frac{1}{4} c_{3} K_{i} r_{i}^{5(\beta-\alpha)}\left(r_{i} / R\right)^{d} \geq 2 .
\end{aligned}
$$

This is a contradiction, and therefore (4.6) is proved.

Write $\tau_{i}$ for $\tau_{B\left(x_{i}, r_{i} / 2\right)}$. Set $M_{i}=\sup _{B\left(x_{i}, r_{i}\right)} u(x)$. Let $E$ be a compact subset of $B\left(x_{i}, r_{i} / 4\right) \backslash A_{i}$ such that $|E| /\left|B\left(x_{i}, r_{i} / 4\right)\right| \geq \frac{1}{2}$. In view of (4.6) such a choice is possible. Let

We have

$$
p_{i}=\mathbb{P}^{x_{i}}\left(T_{E}<\tau_{i}\right) .
$$

$$
\begin{aligned}
K_{i}=u & \left(x_{i}\right)=\mathbb{E}^{x_{i}}\left[u\left(X_{T_{E} \wedge \tau_{i}}\right) ; T_{E}<\tau_{i}\right] \\
& +\mathbb{E}^{x_{i}}\left[u\left(X_{T_{E} \wedge \tau_{i}}\right) ; T_{E} \geq \tau_{i}, X_{\tau_{i}} \in B\left(x_{i}, r_{i}\right)\right] \\
& +\mathbb{E}^{x_{i}}\left[u\left(X_{T_{E} \wedge \tau_{i}}\right) ; T_{E} \geq \tau_{i}, X_{\tau_{i}} \notin B\left(x_{i}, r_{i}\right)\right] .
\end{aligned}
$$

Since $E \subset B\left(x_{i}, r_{i} / 4\right) \backslash A_{i}$ is compact, the first term is bounded above by

$$
K_{i} r_{i}^{4(\beta-\alpha)} \mathbb{P}^{x_{i}}\left(T_{E}<\tau_{i}\right) \leq K_{i} r_{i}^{4(\beta-\alpha)} .
$$

The second term is bounded above by

$$
M_{i}\left(1-p_{i}\right) .
$$

We turn to the third term. Inequality (4.6) implies in particular that there exists $y_{i} \in B\left(x_{i}, r_{i} / 4\right)$ with $u\left(y_{i}\right) \leq K_{i} r_{i}^{4(\beta-\alpha)}$. We then have, using Proposition 3.5.

$$
\begin{aligned}
K_{i} r_{i}^{4(\beta-\alpha)} & \geq u\left(y_{i}\right) \geq \mathbb{E}^{y_{i}}\left[u\left(X_{\tau_{i}}\right) ; X_{\tau_{i}} \notin B\left(x_{i}, r_{i}\right)\right] \\
& \geq c_{5} r_{i}^{2(\beta-\alpha)} \mathbb{E}^{x_{i}}\left[u\left(X_{\tau_{i}}\right) ; X_{\tau_{i}} \notin B\left(x_{i}, r_{i}\right)\right] .
\end{aligned}
$$


Using (4.8), the third term on the right of 4.7) is bounded above by

$$
c_{5}^{-1} r_{i}^{2(\alpha-\beta)} K_{i} r_{i}^{4(\beta-\alpha)}=c_{6} K_{i} r_{i}^{2(\beta-\alpha)} .
$$

Substituting in (4.7), we get

$$
K_{i} \leq K_{i} r_{i}^{4(\beta-\alpha)}+M_{i}\left(1-p_{i}\right)+c_{6} K_{i} r_{i}^{2(\beta-\alpha)} .
$$

Rearranging,

$$
M_{i} \geq K_{i}\left(\frac{1-r_{i}^{4(\beta-\alpha)}-c_{6} r_{i}^{2(\beta-\alpha)}}{1-p_{i}}\right) .
$$

By 4.3.

$$
p_{i} \geq c_{7} c_{3} r_{i}^{\beta-\alpha} .
$$

Since $r_{i} \leq c_{2} R \leq c_{2}$, if we choose $c_{2}$ small enough, then

$$
r_{i}^{4(\beta-\alpha)}+c_{6} r_{i}^{2(\beta-\alpha)} \leq \frac{1}{2} c_{7} c_{3} r_{i}^{\beta-\alpha}
$$

for all $i$. Therefore

$$
M_{i} \geq K_{i}\left(\frac{1-\frac{1}{2} p_{i}}{1-p_{i}}\right)>\left(1+\frac{p_{i}}{2}\right) K_{i} .
$$

Using the definition of $M_{i}$ and (4.11), there exists a point $x_{i+1} \in \overline{B\left(x_{i}, r_{i}\right)} \subset$ $B\left(x_{i}, 2 r_{i}\right)$ such that

$$
K_{i+1}=u\left(x_{i+1}\right) \geq K_{i}\left(1+c_{7} c_{3} r_{i}^{\beta-\alpha} / 2\right) .
$$

Taking logarithms and writing

$$
\log K_{i+1}=\log K_{1}+\sum_{j=1}^{i}\left[\log K_{j+1}-\log K_{j}\right]
$$

we have

$$
\begin{aligned}
\log \left(K_{i+1}\right) & \geq \log K_{1}+\sum_{j=1}^{i} \log \left(1+c_{7} c_{3} r_{j}^{\beta-\alpha} / 2\right) \\
& \geq \log K_{1}+c_{8} \sum_{j=1}^{i} r_{j}^{\beta-\alpha} \\
& =\log K_{1}+R c_{2} c_{8} \sum_{j=1}^{i} j^{-\rho(\beta-\alpha)} \\
& \geq \log K_{1}+R c_{2} c_{4}(i+1)^{1-\rho(\beta-\alpha)},
\end{aligned}
$$

and hence (4.5) holds for $i+1$ provided we choose $c_{2}$ small enough so that (4.2) and (4.12) hold. The theorem has thus been proved for $R<1$.

For $R \geq 1$ we use a standard chain of balls argument. Given any two points $x, y \in B\left(z_{0}, R / 2\right)$, we can find $N$ balls $B_{1} \ldots, B_{N}$ of radius $\frac{1}{2}$ such that $x$ is the center of $B_{1}, y$ is the center of $B_{N}$, the centers of $B_{i}$ and $B_{i+1}$ lie within $\frac{1}{4}$ of each other for each $i$, and the center of $B_{i}$ lies in $B\left(z_{0}, R / 2\right)$ for each $i$. Moreover, the number of balls $N$ depends only on $R$. We then apply the Harnack inequality that we proved above $N$ times to derive $u(x) \leq c_{9}^{N} u(y)$.

Remark 4.2. If one keeps careful track of the constants, one sees that $\bar{\kappa}$ grows at most polynomially in $1 / R$ as $R \rightarrow 0$. See also Proposition 5.1 
Remark 4.3. We do not know if the condition $\beta-\alpha<1$ can be weakened. It would be very interesting to either weaken this condition or find an example showing it is necessary.

Remark 4.4. Must a function that is bounded in $\mathbb{R}^{d}$ and is harmonic in a ball be continuous in the ball? This is the case for nondegenerate diffusions and stable-like jump processes (i.e., $\alpha=\beta$ ), but we do not know the answer to this question in the variable order case. Continuity appears to be a less robust property than the Harnack inequality.

\section{EXAMPLES}

In Theorem 4.1 we allowed the constant $\bar{\kappa}$ to depend on $R$. This is necessary, as the following proposition shows. To see the idea behind the proof, consider $\left(V_{t}^{1}, V_{t}^{2}\right)$, where $V_{t}^{1}$ is a one-dimensional symmetric stable process of order $\beta$ and $V_{t}^{2}$ is a one-dimensional symmetric stable process of order $\alpha$. If $\beta>\alpha$, then over short distances the first component moves much faster than the second. However $\left(V_{t}^{1}, V_{t}^{2}\right)$ does not satisfy Assumption 2.1(a), and so we must use a more complicated example. See Ber96 for information on Lévy processes.

Proposition 5.1. Let $0<\alpha<\beta<2$. There exists a function $n(x, h)$ satisfying Assumption [2.1] with the following property.

- For $R<1$ there exist functions $u_{R}$ that are nonnegative and harmonic on $B(0, R)$ and points $x_{R}, y_{R} \in B(0, R / 2)$ such that $u_{R}\left(y_{R}\right) / u_{R}\left(x_{R}\right) \rightarrow \infty$ as $R \rightarrow 0$.

Proof. Observe that $\lim _{a \rightarrow 1}\left(a-2+\frac{1}{a}\right) /(1-a)=0$. Choose $a<1$ sufficiently close to 1 so that $\beta>\left(a-2+\frac{1}{a}\right) /(1-a)$. Take $a$ closer to 1 if necessary so that $a \beta+a-1>\alpha$. Some algebra shows that $\beta+1-\frac{1}{a}>a \beta+a-1$. We now choose $\gamma$ such that $\beta+1-\frac{1}{a}>\gamma>a \beta+a-1$. Since $a<1$, then $\gamma<\beta$; by our choice of $a$, we see that $\gamma>\alpha$.

Let

$$
A=\left\{\left(x_{1}, x_{2}\right):\left|x_{2}\right|>\left|x_{1}\right|^{a},\left|x_{2}\right|<1\right\} .
$$

We define a Lévy process $X_{t}=\left(X_{t}^{1}, X_{t}^{2}\right)$ by specifying that there is no Gaussian component, no drift, and the Lévy measure is given by $n(d h)=n(h) d h$, where

$$
n(h)=\frac{1}{|h|^{2+\alpha}}+\frac{1_{A}(h)}{|h|^{2+\beta}} .
$$

If we set $n(x, h)=n(h)$ for all $x$, clearly Assumption 2.1 holds.

Let $D_{R}=[-R, R]^{2}, x_{R}=(-R / 4,0), y_{R}=(R / 4,0)$. Define $u_{R}\left(x_{1}, x_{2}\right)$ on $D_{R}^{c}$ to be 1 if $x_{1}>0$ and 0 otherwise. Define $u_{R}$ inside $D_{R}$ by $u_{R}(x)=\mathbb{E}^{x} u_{R}\left(X_{\tau_{D_{R}}}\right)$. Then $u_{R}$ is harmonic in $B(0, R)$.

We will show that

$$
\mathbb{P}\left(\sup _{s \leq R^{\gamma}}\left|X_{s}^{2}\right|<R\right) \rightarrow 0 \quad \text { as } R \rightarrow 0
$$

and

$$
\mathbb{P}\left(\sup _{s \leq R^{\gamma}}\left|X_{s}^{1}\right|>R / 4\right) \rightarrow 0 \quad \text { as } R \rightarrow 0
$$


(5.1) says that for $R$ small, $\left|X_{t}^{2}\right|$ is very likely to have exceeded $R$ by time $R^{\gamma}$; hence $\tau_{D_{R}} \leq R^{\gamma}$ with high probability. (5.2) says that by time $\tau_{D_{R}}$ the process $X_{t}^{1}$ is unlikely to have moved as far as $R / 4$. Consequently

$$
\begin{aligned}
& \mathbb{P}^{x_{R}}\left(X_{\tau_{D_{R}}}^{1}>0\right) \rightarrow 0 \quad \text { as } R \rightarrow 0, \\
& \mathbb{P}^{y_{R}}\left(X_{\tau_{D_{R}}}^{1}>0\right) \rightarrow 1 \quad \text { as } R \rightarrow 0 .
\end{aligned}
$$

This shows that $u_{R}\left(x_{R}\right) \rightarrow 0$ and $u_{R}\left(y_{R}\right) \rightarrow 1$; hence $u_{R}\left(y_{R}\right) / u_{R}\left(x_{R}\right) \rightarrow \infty$ as $R \rightarrow 0$.

We now prove (5.1) and (5.2). Write $h=\left(h_{1}, h_{2}\right)$. Since $a<1$, then for $h_{2} \in[2 R, 3 R]$ and $h_{1} \in\left(0,\left|h_{2}\right|^{1 / a}\right]$ we have that $|h|$ is comparable to $h_{2}$. We calculate

$$
\begin{aligned}
I_{1}(R) & =\int_{A \cap\left(\left|h_{2}\right|>2 R\right)} n(d h) \geq 4 \int_{2 R}^{1} \int_{0}^{\left|h_{2}\right|^{1 / a}} \frac{1}{|h|^{2+\beta}} d h_{1} d h_{2} \\
& \geq 4 \int_{2 R}^{3 R} \int_{0}^{\left|h_{2}\right|^{1 / a}} \frac{1}{|h|^{2+\beta}} d h_{1} d h_{2} \geq c_{1} R^{\frac{1}{a}-1-\beta} .
\end{aligned}
$$

The number of times that $\Delta X_{s} \in A \cap\left(\left|h_{2}\right|>2 R\right)$ for $s \leq t$ is a Poisson random variable with parameter greater than $t I_{1}(R)$. By our choice of $\gamma, R^{\gamma} I_{1}(R) \rightarrow \infty$ as $R \rightarrow 0$. Hence the probability that there are no jumps with $\Delta X_{s}$ in $A \cap\left(\left|h_{2}\right|>2 R\right)$ by time $R^{\gamma}$ tends to 0 as $R \rightarrow 0$. But if $\Delta X_{s} \in A \cap\left(\left|h_{2}\right|>2 R\right)$ for some $s \leq R^{\gamma}$, then $\left|X_{s}^{2}\right|$ will exceed $R$. This proves (5.1).

We turn to (5.2). We can write $X_{t}=Y_{t}+Z_{t}$, where $Y$ and $Z$ are independent Lévy processes, $Y$ has Lévy measure $n_{Y}(d h)=|h|^{-(2+\alpha)} d h$, and $Z$ has Lévy measure $n_{Z}(d h)=1_{A}(h)|h|^{-(2+\beta)} d h$.

By scaling and the fact that $\gamma>\alpha$,

$$
\mathbb{P}\left(\sup _{s \leq R^{\gamma}}\left|Y_{s}\right|>R / 8\right) \rightarrow 0 \quad \text { as } R \rightarrow 0 .
$$

We calculate

$$
\begin{aligned}
I_{2}(R) & =\int_{A \cap\left(\left|h_{1}\right|>R / 16\right)} n_{Z}(d h) \\
& \leq 4 \int_{R / 16}^{1} \int_{\left|h_{1}\right|^{a}}^{1} \frac{1}{|h|^{2+\beta}} d h_{2} d h_{1} \\
& \leq 4 \int_{R / 16}^{1} \int_{\left|h_{1}\right|^{a}}^{1} \frac{1}{\left|h_{2}\right|^{2+\beta}} d h_{2} d h_{1} \\
& \leq c_{2} R^{1-a-a \beta}
\end{aligned}
$$

and

$$
I_{3}(R)=4 \int_{0}^{R} \int_{\left|h_{1}\right|^{a}}^{1} \frac{\left(h_{1}\right)^{2}}{|h|^{2+\beta}} d h_{2} d h_{1} \leq c_{3} R^{3-a-a \beta} .
$$

The expected number of times $\Delta Z_{s}$ is in $A \cap\left(\left|h_{1}\right|>R / 16\right)$ for $s \leq R^{\gamma}$ is $R^{\gamma} I_{2}(R)$, which tends to 0 as $R \rightarrow 0$ by our choice of $\gamma$. Therefore

$$
\mathbb{P}\left(\left|\Delta Z_{s}^{1}\right|>R / 16 \text { for some } s \leq R^{\gamma}\right) \rightarrow 0 \quad \text { as } R \rightarrow 0 .
$$


Let $W_{t}$ be the process $Z_{t}$ with all jumps such that $\Delta Z_{s}$ is in $A \cap\left(\left|h_{1}\right|>R / 16\right)$ removed, that is,

$$
W_{t}=Z_{t}-\sum_{s \leq t} \Delta Z_{s} 1_{A \cap\left(\left|h_{1}\right|>R / 16\right)}\left(\Delta Z_{s}\right) .
$$

Then $W_{t}$ is the Lévy process with Lévy measure

$$
n_{W}(d h)=\frac{1_{A \cap\left(\left|h_{1}\right| \leq R / 16\right)}(h)}{|h|^{2+\beta}} d h .
$$

Since a Lévy process with bounded jumps has moments of all orders and $W$ has no drift component, then $W_{t}^{1}$ is a martingale. By Doob's inequality,

$$
\mathbb{P}\left(\sup _{s \leq R^{\gamma}}\left|W_{s}^{1}\right|>R / 16\right) \leq 4 \frac{\mathbb{E}\left(W_{R^{\gamma}}^{1}\right)^{2}}{(R / 16)^{2}} .
$$

But $\mathbb{E}\left(W_{t}^{1}\right)^{2}=t I_{3}(R)$, and so by our choice of $\gamma$ we have $\mathbb{E}\left(W_{R^{\gamma}}^{1}\right)^{2} / R^{2} \rightarrow 0$ as $R \rightarrow 0$. Therefore

$$
\mathbb{P}\left(\sup _{s \leq R^{\gamma}}\left|W_{s}^{1}\right|>R / 16\right) \rightarrow 0 \quad \text { as } R \rightarrow 0 .
$$

Putting (5.3), (5.4), and (5.5) together gives (5.2).

The following example shows that a hypothesis along the lines of Assumption 2.1(d) is necessary for a Harnack inequality to hold.

Proposition 5.2. There exists a function $n(x, h)$ satisfying Assumptions[2.1(a)-(c) (but not (d)) for which the Harnack inequality fails for the corresponding operator.

Proof. We work in two dimensions. Let $B=B(0,1)$, let $y_{0}=(1 / 8,0)$ and for $m \geq 4$ let $x_{m}=\left(-1 / 8,2^{-m}\right), z_{m}=\left(16,2^{-m}\right), C_{m}=B\left(x_{m}, 2^{-m-4}\right)$, and $E_{m}=$ $B\left(z_{m}, 2^{-m-4}\right)$. Define

$$
n(x, h)=|h|^{-d-\alpha} 1_{(|h| \leq 3)}+\sum_{m=4}^{\infty} 1_{C_{m}}(x) 1_{E_{m}}(x+h) .
$$

It is clear that $n(x, h)$ satisfies Assumptions 2.1 (a)-(c), because the $C_{m}$ are disjoint and the $E_{m}$ are disjoint. It is also not hard to see that there is a unique solution to the martingale problem for $\mathcal{L}$, because $n$ differs from the Lévy kernel of a symmetric stable process only in the jumps of size larger than 3, see Kom84.

Next we show that $\mathbb{P}^{y_{0}}\left(T_{C_{m}}<\tau_{B}\right)$ is small when $m$ is large. Note that Lemma 3.4 does not use Assumption 2.1(d), and therefore $\mathbb{E}^{y_{0}} \tau_{B} \leq c_{1}<\infty$. Fix $m$, let $\varepsilon=2^{-m-4}$, let $g(x)=\left|x-x_{m}\right|^{-d-\alpha}$, let $\varphi$ be a nonnegative $C^{\infty}$ function with support in $B(0,1 / 2)$ whose integral is 1 , let $\varphi_{\varepsilon}(x)=\varepsilon^{-d} \varphi(x / \varepsilon)$, and let $f_{\varepsilon}=g * \varphi_{\varepsilon}$. Let $n_{0}(x, h)=|h|^{-d-\alpha}$ and let $\mathcal{L}_{0}$ be the operator corresponding to $n_{0}$. Then $f_{\varepsilon} \geq c_{2} \varepsilon^{-\alpha-d}$ on $C_{m}$ and $f_{\varepsilon} \in C^{\infty}$. It is well known that $\mathcal{L}_{0} f_{\varepsilon}(x)=-c_{3} \varphi_{\varepsilon}\left(x-x_{m}\right)$, and hence $\mathcal{L}_{0} f_{\varepsilon}(x)=0$ for $x \notin C_{m}$. It is also easy to check that $f_{\varepsilon}\left(y_{0}\right) \leq c_{4}$ and $\left|\mathcal{L} f_{\varepsilon}(x)-\mathcal{L}_{0} f_{\varepsilon}(x)\right| \leq c_{5}$ if $x \in B \backslash C_{m}$. Therefore $\mathcal{L} f_{\varepsilon}(x) \leq c_{5}$ for $x \in B \backslash C_{m}$. Since $\mathbb{P}^{y_{0}}$ is a solution to the martingale problem for $\mathcal{L}$, we have

$$
\begin{aligned}
\mathbb{E}^{y_{0}} f_{\varepsilon}\left(X_{T_{C_{m}} \wedge \tau_{B}}\right)-f_{\varepsilon}\left(y_{0}\right) & =\mathbb{E}^{y_{0}} \int_{0}^{T_{C_{m}} \wedge \tau_{B}} \mathcal{L} f_{\varepsilon}\left(X_{s}\right) d s \\
& \leq c_{5} \mathbb{E}^{y_{0}} \tau_{B} \leq c_{1} c_{5} .
\end{aligned}
$$


Therefore

$$
c_{2} \varepsilon^{-\alpha-d} \mathbb{P}^{y_{0}}\left(T_{C_{m}}<\tau_{B}\right) \leq \mathbb{E}^{y_{0}} f_{\varepsilon}\left(X_{T_{C_{m}} \wedge \tau_{B}}\right) \leq c_{1} c_{5}+c_{4} .
$$

Thus $\mathbb{P}^{y_{0}}\left(T_{C_{m}}<\tau_{B}\right)$ will be small if $m$ is large.

Now suppose that the Harnack inequality did hold for nonnegative functions that are harmonic in $B$, that is, suppose there exists $c_{6}$ such that

$$
u(x) \leq c_{6} u(y), \quad x, y \in B(0,1 / 2),
$$

whenever $u$ is bounded in $\mathbb{R}^{d}$ and nonnegative and harmonic in $B$. Let

$$
u_{m}(x)=\mathbb{E}^{x}\left[1_{E_{m}}\left(X_{\tau_{B}}\right)\right] .
$$

This function is bounded, nonnegative, and harmonic in $B$. Note the only way that $X_{\tau_{B}}$ can be in $E_{m}$ is if $X_{\tau_{B}-}$ is in $C_{m}$. We then have

$$
\begin{aligned}
& u_{m}\left(y_{0}\right)=\mathbb{E}^{y_{0}}\left[1_{E_{m}}\left(X_{\tau_{B}}\right) ; T_{C_{m}}<\tau_{B}\right]
\end{aligned}
$$

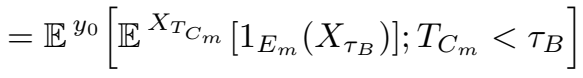

$$
\begin{aligned}
& =\mathbb{E}^{y_{0}}\left[u_{m}\left(X_{T_{C_{m}}}\right) ; T_{C_{m}}<\tau_{B}\right] \\
& \leq c_{6} u_{m}\left(x_{m}\right) \mathbb{P}^{y_{0}}\left(T_{C_{m}}<\tau_{B}\right),
\end{aligned}
$$

where we used the assumption that a Harnack inequality holds to get the last inequality. But then

$$
\frac{u_{m}\left(x_{m}\right)}{u_{m}\left(y_{0}\right)} \geq \frac{1}{c_{6} \mathbb{P}^{y_{0}}\left(T_{C_{m}}<\tau_{B}\right)},
$$

which can be made arbitrarily large if we take $m$ large enough. This is a contradiction, and therefore the Harnack inequality cannot hold.

\section{ACKNOWLEDGMENTS}

We would like to thank Martin Barlow for helpful conversations on the subject of this paper.

\section{REFERENCES}

[Bas88] R. F. Bass. Uniqueness in law for pure jump Markov processes. Probab. Theory Related Fields, 79(2):271-287, 1988. MR 89h:60118

[BBG00] M.T. Barlow, R.F. Bass, and C. Gui. The Liouville property and a conjecture of De Giorgi. Comm. Pure Appl. Math., 53(8):1007-1038, 2000. MR 2001m:35095

[Ber96] J. Bertoin. Lévy Processes. Cambridge University Press, Cambridge, 1996. MR 98e:60117

[BG68] R. M. Blumenthal and R. K. Getoor. Markov Processes and Potential Theory. Pure and Applied Mathematics, Vol. 29. Academic Press, New York, 1968. MR 41:9348

[BL02a] R.F. Bass and D.A. Levin. Harnack inequalities for jump processes. Potential Anal., 17(4):375-388, 2002. MR 2003e:60194

[BL02b] R.F. Bass and D.A. Levin. Transition probabilities for symmetric jump processes. Trans. Amer. Math. Soc., 354(7):2933-2953, 2002. MR 2002m:60132

[BSS02a] K. Bogdan, A. Stós, and P. Sztonyk. Harnack inequality for stable processes on $d$-sets. Studia Math. 158(2):163-198, 2003. MR 2013738

[BSS02b] K. Bogdan, A. Stós, and P. Sztonyk. Potential theory for Lévy stable processes. Bull. Polish Acad. Sci. Math. 50(3):361-372, 2002. MR 2003m:60125

[CK03] Z.-Q. Chen and T. Kumagai. Heat kernel estimates for stable-like processes on $d$-sets. Stochastic Processes Appl. 108(1):27-62, 2003. MR 2008600

[FS86] E.B. Fabes and D.W Stroock. A new proof of Moser's parabolic Harnack inequality via the old ideas of Nash. Arch. Mech. Rat. Anal., 96:327-338, 1986. MR 88b:35037 
[Hoh95] W. Hoh. Pseudodifferential operators with negative definite symbols and the martingale problem. Stochastics Stochastics Rep., 55(3-4):225-252, 1995. MR 97c:35225

[Kas03] M. Kassmann. On regularity for Beurling-Deny type Dirichlet forms. Potential Analysis, 19(1):69-87, 2003. MR 2004a:31005

[Kom84] T. Komatsu. On the martingale problem for generators of stable processes with perturbations. Osaka J. Math., 21(1):113-132, 1984. MR 86e:60060

[KS79] N. V. Krylov and M. V. Safonov. An estimate for the probability of a diffusion process hitting a set of positive measure. Dokl. Akad. Nauk SSSR, 245(1):18-20, 1979; English transl., Soviet Math. Dokl. 20(1):253-255, 1979. MR 80b:60101

[KS80] N. V. Krylov and M. V. Safonov. A property of the solutions of parabolic equations with measurable coefficients. Izv. Akad. Nauk SSSR Ser. Mat., 44(1):161-175, 239, 1980; English transl., Math. USSR-Izv. 16(1):151-164, 1981. MR 83c:35059

[Mos61] J. Moser. On Harnack's theorem for elliptic differential equations. Comm. Pure Appl. Math., 14:577-591, 1961. MR 28:2356

[Sko65] A. V. Skorokhod. Studies in the Theory of Random Processes. Addison-Wesley Publishing Co., Inc., Reading, Mass., 1965. MR 32:3082b

[SV04] R. Song and Z. Vondracek. Harnack inequality for some classes of Markov processes. Math. Z. 246(1-2):177-202, 2004.

[SV79] D. W. Stroock and S. R. S. Varadhan. Multidimensional Diffusion Processes, Volume 233 of Grundlehren der Mathematischen Wissenschaften. Springer-Verlag, Berlin, 1979. MR 81f:60108

Department of Mathematics, University of Connecticut, Storrs, Connecticut 062693009

E-mail address: bass@math.uconn.edu

Department of Mathematics, University of Connecticut, Storrs, Connecticut 062693009 - And Institut für Angewandte Mathematik, Universität Bonn, Beringstrasse 6, D-53115 Bonn, Germany

E-mail address: kassmann@math.uconn.edu 